\title{
EL LIBRO DE PRIVILEGIOS DE ELCHE: ESTUDIO I
}

\author{
Mㄹ Luisa Cabanes Catalá \\ Universidad de Alicante
}

El llamado "Códice de Elche" es en realidad un libro de privilegios que no sido estudiado, ni publicado en su totalidad, si bien algunos de sus documentos figuran en colecciones diplomáticas publicadas o en historias (1), existiendo,además, un pequeño trabajo sobre los sistemas de datación usados en él (2). Abordamos, aquí, el estudio del Libro de privilegios de Elche (3) que se articula en dos grandes apartados: el primero que comprende los aspectos codicológicos y paleográficos y el segundo que recogerá los diplomáticos (4) según el siguiente esquema:

I.- Estudio codicológico y paleográfico

1.- Descripción externa

2.- Historia del manuscrito y bibliografía

II.- Aspectos diplomáticos

1.- Clasificación documental

2.- Documentos reales

3.- Documentos señoriales

4.- De autoridades delegadas

5.- Notariales

6.- Concejiles 


\section{I.- ESTUDIO CODICOLÓGICO Y PALEOGRÁFICO}

Para el estudio codicológico del "Libro de Privilegios de Elche", hemos usado el esquema de Armando Petrucci en La descrizioni del manoscritto. Storia, problemi, modelli (5).

De acuerdo, pues, con el mismo la pauta a seguir en este estudio es la siguiente:

1.- Descripción externa

1.1.- Signatura y materia

1.2.- Fecha

1.3.- Preparación y dimensiones

1.4.- Estructura

1.5.- Foliación

1.6.- Reclamos

1.7.- La escritura

1.8.- La ornamentación

1.9.- La encuadernación

2.- Historia del Códice

3.- Bibliografía

\section{1.- Signatura y materia}

El Libro de Privilegios de Elche, no tiene una signatura específica dentro del Archivo Histórico Municipal de Elche, se le conoce habitualmente con la denominación de "códice", pero por nuestra parte preferimos la del Libro de Privilegios, ya que dentro de ese tipo hay que incluirlo.

Como es habitual, está confeccionado en pergamino, que, posiblemente, debió prepararse en la misma población, según se deduce de lo irregular de su acabado:

* unas hojas presentan mayor grosor que otras

* hay una notable diferencia entre la parte que estuvo en contacto con la carne, que suele ser bastante blanca, y la que estuvo en contacto con el 
pelo, que adquiere una gran variedad de tonalidades, desde el amarillo paja hasta un marrón oscuro, pasando por tonalidades grisáceas.

\section{2.- Fecha}

Uno de los problemas más interesantes que presenta el Libro de Privilegios de Elche es la de su datación.

El manuscrito que ha llegado hasta nosotros es un códice facticio, ya que se aprecian dos partes cláramente diferenciadas por:

* existencia de dos portadas

* aparición de nuevos elementos decorativos, en lo que denominamos segunda parte, respecto a la primera, por ejemplo, de tipo heráldico.

* el uso de iniciales de gran tamaño en la segunda parte, llegando incluso a ocupar la altura completa de la caja de escritura

* diferente distribución del texto en las dos parte

* distinta caja de escritura

* existencia de dos colofones

Entre una parte y otra, las hojas que quedaron en blanco del cuaderno fueron, porteriormente, usadas para copiar otros documentos, estando uno de ellos incompleto sin motivo aparente.

Lo que consideramos primera parte, en su clausura indica que se inició su elaboración en 1316, concluyendola en 1312. Se hizo con los documento que tenía Domingo Pascual, jurado de Elche, para su custodia, siendo en total setenta y cuatro, los cuales ocuparon setenta y seis folios. Hace constar, asímismo, que los autores de los instrumentos fueron los reyes de Castilla, los señores de Elche, el procurador del Reino, haciendo, también, expresa mención de las protesta elevadas por los ilicitanos ante las autoridades competentes. El autor material sería Francesch Maella, el cual regía la escribanía de la curia por Berenguer de Puigmnolto, realizándolo por encargo de Domingo Pascual y Diego Alfonso: 
"Sig (signo) num de mi Francesch Mealla, notari publich de Elx e escriva del Consell de est mateix loch per honrat En Berenguer de Puigmolto, qui vist los originals privilegis e cartes del sennor rey, partida d'ells son en pergami escrites e segellades ab segells pendents en cordes reals de seda de diverses colors, posades ab lo segell del sennor rey, entre les quals ha una carta, escripta en paper, del noble N'Acard de Mur, procurador d'esta partida, e segellada el dors ab lo segell usat de la dita procuracio. Et vistes, otrosi, los privilegis originals, cartes del rey de Castella e de lurs mullers, e de lurs fills, et de don Johan, fill del infant don Manuel et de la dona sa mare, partida d'ells segellats en cordes pendents et partida en paper escriptes e segellades al dors. Et, vistes los altres cartes e protestacions de contrast, qu'el Concell d'est loc avia ab alcunes persones, los quals privilegis, cartes e protestacions son per totes setanta quatre, et son escrites en setenta e sis cartes d'est libre, en lo començament d'ell et començant d'ell et comenca lo dit traslat del dit libre "Axi es translat et com" et fenex $M^{\circ}$ CCCXVI.

Et ben e diligentment examinats e comprovats, de paraula a paraula,e de letra a letra, e de punt a punt, los quals privilegis, cartes e protestacions, ço es, los dits originals foren tornats en la caxa del Consell //78 de Elix, que tenia en son poder En Domingo Pasqual, jurat. Et, otrosi, regent yo la escrivania de la cort del dit loc, per lo dit En Berenguer de Puigmolto, de manament del dit justicia, la sua auctoritat e decret, y escrivi, et a pregaries e manament del dit En Domingo Pasqual et En Diego Alfonso, jurats de ElX, lo meu signe acostumat y pose en testimoni de veritat, e lo acloy diIluns XII dies de octubre anno Domini Mo CCC vicesimo primo"(6)

La segunda parte, presenta el cierre en muy mal estado, lo que hace imposible su lectura completa. Sin embargo, de la parte que permite la misma, se indica que se copiaron 36 documentos de Jaime II y dos de los reyes de Castilla, sobre 36 folios mayores, realizándose la copia al final de un libro de privilegios que ya tenía el Consell de Elche, igualmente sobre pergamino y, pensamos, es, lo que llamamos, primera parte (7).

El primer documento se data en de 1256 y el último, corresponde a un traslado de un documento del infante Ramón Berenguer, sin fecha. Sin embargo, debe ser anterior a 1327, momento en que muere Jaime II, tanto por el tipo de letra como porque en la clausura de la segunda parte se habla del monarca como si aún estuviera reinando:

“... qui vist los originals privilegis et cartes del senyor rey ..." (8) 
Es decir, la cronología de realización del Libro de Privilegios nos lo situaría una fecha anterior a 1327, año de la muerte de Jaime II. Esta se encuentra en completa oposición a la datación tradicional que lo fecha en 1523, basándose en una referencia del Consell del 26 de septiembre de dicho año:

Disapte XXVI dies del mes de setembre, any de la Nativitat de Nostre Senyor MDXXIII.

Item, lo magnifich Consell, atenent en dies pasats sta feta hordenacio, que de totos los privillegis que te la dita vila, sien mesos e traslladats en un libre de pergami fet, e may sis stat aquell fet fins a huy, per allo dit magnifich Consell dona facultat e comisio al justicia, jurats e sindich de la dita vila, que aquells, 10 mes prest que puxen, ajen un bon scrivent pera que faca (sic) dit trellat de tots los privilegis que te la dita vila y lo dit Consell en paper y en pergami, pagan-li al dit scrivent que axi fara lo dit trellat de privilegis sos justs treballs per lo clavari del dit Consell e li sia posat en conte de paga al dit clavari (9)

A esta datación tradicional se opone, además, de los argumentos ya expuestos:

- el tipo de escritura "gótica textual" que es la usual del siglo XIV y XV, no la del siglo XVI

- la decoración de las iniciales, sencilla pero, plenamente, incardinada en la centuria del Trescientos, por lo tanto gótica, y no en la del Quinientos

- no hay ningún documento posterior a 1321, cuando entre esa fecha y 1523 la villa de Elche tuvo varios señores (perteneció a la Corona, estuvo enfeudada a la ciudad de Barcelona, nuevamente, pasó a la Corona, luego a los duques de Maqueda, etc.) y por lo tanto recibió documentos importantes, algunos de los cuales se conservan hoy en su archivo

- la mención, en el colofón de la segunda parte, de Jaime II como reinando en ese momento $y$, por lo tanto, vivo.

Por todo ello, pensamos que el Libro de Privilegios, tuvo que ser confeccionado en el siglo XIV y antes de 1327 , momento de la muerte de Jaime II, justificando la realización del mismo al haberse incorporado Elche a la Corona de Aragón bajo el citado monarca y necesitando los ilicitanos tener todos los privilegios concedidos spor los monarcas y señores anterio- 
res reunidos, así como los documentos que el consell ilicitano consideraba importante para, si llegaba el caso, poderlos presentar.

\subsection{Preparación y dimensiones}

Las hojas del códice han sido preparadas antes de ser escritas. Las hojas de los cuadernos que componen la primera parte, las perforaciones se realizadaron por medio de un compas, que marcó la distancia entre las pautas, realizádose para todo un cuaderno a la vez, aunque no están alineadas, ya que, cuando se trazó la caja de escritura y el pautado - que se hizo hoja a hoja -, quedaron fuera, formando una línea oblicua.

La preparación se ha llevado a efecto por la parte de la carne, el instrumento ha sido un puzón, que en ocasiones se podría haber mojado ligéramente de tinta, ya que en algunas hojas presentan en el pautado huella de tinta de tono marrón.

La segunda parte, con una preparación semejante, aunque a diferencia de la primera no se ha hecho para todo el cuaderno sino hoja por hoja (Lams. 1, 2 y 3).

La caja de escritura no tiene unas dimensiones uniformes, ya que oscila entre 160 por $190 \mathrm{~mm}$. y 220 por $155 \mathrm{~mm}$. Asímismo las dimensiones de las hojas no son iguales, si bien predomina el formato de 305 por $220 \mathrm{~mm}$.

El número de líneas es, igualmente, variable entre 32 y 39 , aunque el número más frecuente es de 34 . A modo de resumen y comparación entre caja de escritura, dimensiones de las hojas y número de lineas, puede verse el siguiente cuadro:

\begin{tabular}{rccc} 
Na FOL. & DIMENSIONES HOJA & CAJA ESCRITURA & Nª LINEAS \\
\hline III & $305 \times 215 \mathrm{~mm}$. & & 34 \\
5 & $305 \times 220 \mathrm{~mm}$. & $160 \times 190 \mathrm{~mm}$. & 34 \\
9 & $305 \times 220 \mathrm{~mm}$. & $185 \times 160 \mathrm{~mm}$. & 34 \\
43 & $305 \times 220 \mathrm{~mm}$. & $220 \times 155 \mathrm{~mm}$. & 36 \\
45 & $305 \times 215 \mathrm{~mm}$. & $220 \times 140 \mathrm{~mm}$. & 34 \\
47 & $305 \times 220 \mathrm{~mm}$. & $195 \times 140 \mathrm{~mm}$. & 34 \\
94 & $305 \times 220 \mathrm{~mm}$. & $210 \times 160 \mathrm{~mm}$. & 39 \\
\hline
\end{tabular}




\section{4.- Estructura}

El Libro de Privilegios de Elche, tuvo en su origen 120 folios, de los que sólo conserva 116 - habiendo perdido cuatro - que corresponden al folio VI, 19, 80 y 89. La distribución de los mismos se hace en 17 cuadernos, siendo como es frecuente, variable en su composición, el $1^{\circ}$ y el último, respecto a los demás (Lams. 4 y 5):

$1^{\circ}$ cuaderno: se trata de un ternión $(3+2)$, pero que ha perdido el último folio. Va foliado, con numeración romana, independientemente del resto del libro ya que contiene los índices.

$2^{\circ}$ cuaderno: en su origen fue un cuaternión (4+4). Entre el folio $6 \mathrm{v}$ y el 7 le han añadido los folios 22 y 23, cuya adición sería correcta según los reclamos $y$, posiblemente, se introdujeron, en este cuaderno, cuando se encuadernó en el siglo XVIII

$3^{\circ}$ cuaderno: inicia la foliación continuada y está formado por un cuaternión $(4+4)$

$4^{\circ}$ cuaderno: originariamente debió ser un cuaternión $(3+2)$, si bien en la actualidad ha perdido 3 hojas, las correspondientes a los folios 22 y 23 de los que ya hemos hablado - y el fólio 19

$5^{\circ}$ al $10^{\circ}$ cuaderno: todos cuaterniones y completos (4+4)

11ํㅡ cuaderno: está incompleto, debió ser un cuadernión $(4+3)$, pero ha perdido el último folio

$12^{\circ}$ y $13^{\circ}$ cuadernos: dos cuaterniones completos (4+4). En el 130 se inicia la foliación arábiga y da principio también la tercera parte del códice

14 cuaderno: en prinicipio, debió de ser un cuaternión $(3+4)$ que ha perdido el primer folio. Ello viene atestiguado por una nota de lbarra, en el margen superior derecho, que indica donde completar el documento iniciado.

$15^{\circ}$ y $16^{\circ}$ cuadernos: son dos cuaterniones $(4+4)$

$17^{\circ}$ cuaderno: se trata de un bifolio $(1+1)$ 


\section{5.- Foliación}

Este libro de privilegios, no presenta foliación continuada, ni tampoco correcta. El primer cuaderno, el que lleva el índice de los documentos, va sin foliar. La foliación "continuada" se inicia en el segundo cuaderno. En este hay una anomalía, porque entre el folio $6^{v}$ y 7 se han intercalado dos folios, los que llevan los números 23 y 22 . Este hecho se justifica, tal y como se ha indicado, porque los reclamos exigen la presencia de los citados folios.

Hasta el cuaderno 11ํㅡ, la foliación aparece indicada mediante numeración romana, situada en el ángulo superior derecho y trazados con tinta roja. Desde éste se inicia la doble foliación, romana y arábiga, si bien no coinciden, ya que en ocasiones no tienen presente los folios desaparecidos, mientras que en otro si. Desde nuestro punto de vista ambas son incorrectas, por ello, en la edición se ha foliado, nuevamente, aunque se ha elaborado una tabla de equivalencia. A partir del fólio 98 la romana se tranforma también en arabiga, siendo, posiblemente ambos del siglo XIX.

\section{TABLA DE EQUIVALENCIAS}

\begin{tabular}{ccc} 
FOL. DADA & FOL. ROMANA Y ARABIGA & FOL. ARABIGA \\
\hline I & - & - \\
II & - & - \\
III & - & - \\
IV & - & - \\
V & - & - \\
VI & - & - \\
1 & I & - \\
2 & II & - \\
3 & III & - \\
4 & IIII & - \\
5 & V & - \\
6 & VI & - \\
7 & VII & - \\
8 & VIII & - \\
9 & IX & - \\
10 & $X$ & - \\
11 & XI & - \\
12 & XII & - \\
13 & XIII & -
\end{tabular}


14

15

16

17

18

19

20

21

22

23

24

25

26

27

28

29

30

31

32

33

34

35

36

37

38

39

40

41

42

43

44

45

46

47

48

49

50

51

52

53

54
XIV

XV

XVI

XVII

XVIII

-

$X X I$

XXII

XXIII

XXIV

XXV

$X X V \mid$

XXVII

XXVIII

$X X I X$

$X X X$

$X X X I$

XXXII

$X X X \|$

XXXIV

XXXV

$X X X V I$

XXXVII

XXXVIII

$X X X \mid X$

$X X X X$

$X X X X I$

$X X X X I$

$X X X X I I I$

$X X X X I I I$

$X X X X V$

$X X X X V I$

XXXXVII

XXXXVIII

$X X X X V \| I I$

L

LI

LII

LIII

LIIII
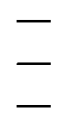

-

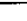




\begin{tabular}{|c|c|c|}
\hline FOL. DADA & FOL. ROMANA Y ARABIGA & FOL. ARABIGA \\
\hline 55 & LV & - \\
\hline 56 & LVI & - \\
\hline 57 & LVII & - \\
\hline 58 & LVIII & - \\
\hline 59 & LVIIII & - \\
\hline 60 & $L X$ & - \\
\hline 61 & LXI & - \\
\hline 61 & LXII & - \\
\hline 63 & LXIII & - \\
\hline 64 & LXIIII & - \\
\hline 65 & LXV & - \\
\hline 66 & LXVI & - \\
\hline 67 & LXVII & - \\
\hline 68 & LXVIII & 一 \\
\hline 69 & LXVIIII & - \\
\hline 70 & LXX & - \\
\hline 71 & LXXI & - \\
\hline 72 & LXXII & - \\
\hline 73 & LXXIII & - \\
\hline 74 & LXXIIII & - \\
\hline 75 & LXXV & - \\
\hline 76 & LXXVI & - \\
\hline 77 & LXXVII & - \\
\hline 78 & LXXVIII & - \\
\hline 79 & LXXVIIII & - \\
\hline 80 & - & - \\
\hline 81 & LXXX & 83 \\
\hline 82 & 81 & 84 \\
\hline 83 & 82 & 85 \\
\hline 84 & 83 & 86 \\
\hline 85 & 84 & 87 \\
\hline 86 & 85 & 88 \\
\hline 87 & 86 & 89 \\
\hline 88 & 87 & 90 \\
\hline 89 & - & - \\
\hline 90 & 88 & 92 \\
\hline 91 & 89 & 93 \\
\hline 92 & 90 & 94 \\
\hline 93 & 91 & 95 \\
\hline 94 & 92 & 96 \\
\hline 95 & 93 & 97 \\
\hline
\end{tabular}




$\begin{array}{rrr}96 & 94 & 98 \\ 97 & 95 & 99 \\ 98 & 96 & 100 \\ 99 & 97 & 101 \\ 100 & 98 & 102 \\ 101 & 99 & 103 \\ 102 & 100 & 104 \\ 103 & 101 & 105 \\ 104 & 102 & 106 \\ 105 & 103 & 107 \\ 106 & 104 & 108 \\ 107 & 105 & 109 \\ 108 & 106 & 110 \\ 109 & 107 & 111 \\ 110 & 108 & 112 \\ 111 & 109 & 113 \\ 112 & 110 & 114 \\ 113 & 111 & 115 \\ 114 & 112 & 116\end{array}$

\section{6. - Los reclamos}

Además de la foliación, el orden de las hojas quedaba garantizado mediante los reclamos. Diferenciamos dos tipos:

- unos son ejecutados con letra de módulo, pequeño y cursivo, que indica la sucesión de las hojas dentro de un cuaderno

- otros usan una letra de módulo mayor, están colocados perpendiculares a las líneas de escritura, van enmarcados y, a veces, por la encuadernación moderna, están, parcialmente, cortados. estos indican el orden de los cuadernos y son los siguientes:

- pedir, fol. $8^{v}$

- sepan, fol. $16^{v}$

- e notari, fol. $24^{v}$

- facer, fol. 32

- d'Entenca, fol. 40"

- tenent, fol. $48^{\circ}$

- costrenner, fol. $56^{\prime}$

- te quanto, fol. $64 \mathrm{v}$ 
- sia a tots, fol. $72 v$

- plebimus, fol. $88 \mathrm{v}$

- significamus, fol. $96 \mathrm{v}$

- solvere, fol. $104 \mathrm{v}$

- latum, fol.110v

El cuaderno décimo primero carece de reclamo.

Los reclamos que indican el orden de los folios son:

-en lo dicto, fol. $\mathrm{l}^{\mathrm{N}}$

-neral, fol IIV

-privilegi, fol. $\| \mathrm{II}^{\mathrm{v}}$

-hellus, fol. $\mathrm{V}^{v}$

-que sacaren, fol. $1^{v}$

-este es traslado, fol. $2^{v}$

-et de todos, fol. $3^{v}$

-siere a la cibdat, fol. $5^{v}$

-villa, fol. $6^{v}$

-que nos den, fol. $22^{v}$

-don Agustín, obispo, fol. $7^{\vee}$

-pora nos, fol. 9

-o de us parientes, fol. $10^{\mathrm{v}}$

-sepan, fol. $17^{\circ}$

-este es traslado, fol. 18

-signum de mi Pere, fol. $25^{v}$

-De mi infante, fol. $26^{\mathrm{v}}$

-Peregrinage, fol. $27^{\circ}$

-nueve, fol. $29^{v}$

-jura, fol. $30^{v}$

-çer, fol. $32 \mathrm{v}$. el, fol. $32^{\mathrm{v}}$

-porque, fol. $35^{v}$

-segnal, fol. $38^{\vee}$

-lomeu. fol. $41^{v}$

-Gil, fol. 42"

-mandaderia, fol. $45^{\vee}$

-gons, fol. $46^{v}$

-dijous, fol. $47^{v}$

-ciones, fol. $49^{\circ}$

-porque, fol. $53^{\mathrm{v}}$

-testes sunt, fol. $54^{v}$

-hoc est, fol. $55^{v}$

-ab este, fol. $56^{v}$

-Domini, fol. $58^{v}$ 


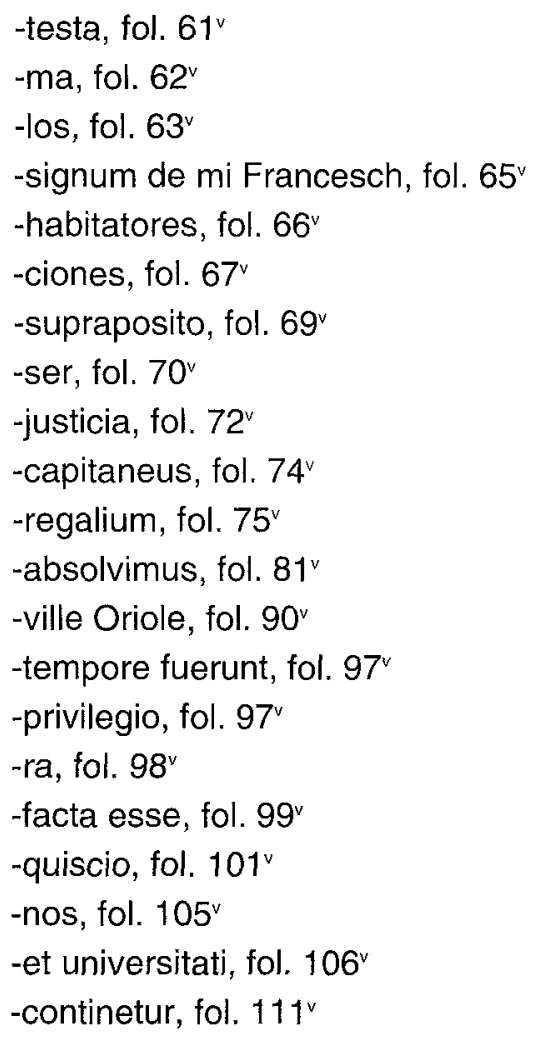

\section{7.- La escritura}

En este libro de privilegios se aprecian varias manos en la ejecución de la obra, todas ellas corresponde al ciclo de la escritura gótica. Las glosas marginales corresponde, en su mayoría, a anotaciones posteriores, escritas en humanística cursiva.

Para la clasificación de las escrituras góticas nos hemos basado en los trabajos de Lieftinck, Canellas, Mateu Ibars, Gasparri, Alvarez, Ruiz Asencio y otros (10). Los tipos de gótica usados son tres:

a.- Gótica textual formata

b.- Gótica textualis

c.- Gótica cursiva aragonesa 


\section{a.- Gótica textual formata}

Aparece sólo en el folio $78^{\vee}$ y copiando un documento del infante don Manuel. Destacamos, especialmente, su alfabeto mayúsculo, semejante al que ofrece Millares (11) para las iniciales de la letra de albalaes; los ojos de las letras se han agrandado de forma desmesurada y en el interior de algunas de ellas, especialmente, la $d$ y la $p$ se han rellenado con decoraciones geométricas.

Los caracteres gráficos de la escritura se encuentran perfectamente marcados:

-ruptura y fractura de los arcos superiores

-bisel en el remate de los alzados

-perfecta observancia de las leyes de Meyer

Finalmente, indicaremos que hace poco uso de las abreviaturas (Lams. 6, 7 y 8)

\section{b.- Gótica textualis}

Con ella se ha escrito la casi totalidad del, Libro de Privilegios, si bien creemos que se puede hablar de tres manos, señalando como características comunes:

-se trata de una escritura menos angulosa que la formata

-sus letras tienen aspecto redondeado

-poco desarrollo de los ástiles ascendentes y descendentes

-observancia de las leyes de Meyer

-uso de pocas abreviaturas

Tres son las manos que escribieron con este tipo, que hemos denominado copista "A", copista "B" y copista "C".

El copista "A", se encargó de escribir desde el folio 1 al 76". Su alfabeto mayúsculo es sencillo, utilizando las formas iniciales para las letras " $c$ " $y$ "m", haciendo uso, también, de minúsculas agrandadas como es el caso de la " $h$ ". Las mayúsculas no suelen estar rellenas, ni llevan trazos decorativos. El alfabeto minúsculo es sencillo, fácil de identificar, destacando, especialmente, la doble grafía de " $r$ ". Es la parte del manuscrito donde se 
han usado mayor número de abreviaturas, tanto las de signo general, que es una línea, como los de signos especiales, letras sobrepuestas o modificaciones literales (Lams. 9, 10 y 11)

El copista " $B$ ", ha ejecutado los folios $78^{\vee}$ y 79 , exceptuando del primer folio citado, el documento del infante don Manuel, que lo escribió otra mano y en distinto tipo de letra. Las mayúsculas utilizadas pertenecen al alfabeto capital en su mayóría, excepto " $d$ " y " $m$ " que son del uncial. Presentan rasgos innecesarios ejecutados con finalidades de adorno. Del alfabeto minúsculo destaca, especialmente, la morfología diferente que adopta la "s". En general, se trata de una escritura algo más tosca que la del copista que hemos llamado "A" (Lams. 12, 13 y 14)

Finalmente, el copista "C" se encargó de la ejecución de lo que hemos considerado segunda parte. Se inicia en el folio 80 y concluye en el 113. Creemos que de los tres es el que ofrece una escritura más firme, mejor ejecutada y más uniforme. Al igual que los copistas "A" y "B", emplea las unciales " $e$ " $\mathrm{y}$ " $\mathrm{m}$ " como mayúsculas, siendo el resto del alfabeto capital y están exentas de rasgos superfluos o de adorno.Destaca de su alfabeto minúsculo la duplicidad de grafías de algunas letras como " $g$ ", " $r$ " y " $s$ ". Respecto al uso de las abreviaturas, este copista apenas las ha utilizado (Lams. 15, 16 y 17)

\section{c.- Gótica cursiva aragonesa}

Se escribieron en este tipo (12) los folios $76^{\vee}$ al $77^{\vee}$ y figura en los dos cierra de cada una de las dos partes señaladas. Es de trazado más rápido que las restantes del códice y como caracteres generales podemos señalar:

- excesiva prolongación de los alzados y caídos de las letras

- el trazo final de " $m$ " tiende a volverse hacía la derecha, siendo más largo que el resto de los trazos

- mayor uso de abreviaturas que en el resto del manuscrito

Algunas características específicas de las letras:

- la "g", desciende por debajo de la línea y luego gira a la izquierda buscando el parelelo con la pauta, llegando a cerrarse 
- la utilización de diferentes tipos de "s" según el lugar que ocupa en la palabra, como es usual en este tipo de letra, así se usa la alta en principio y en medio de palabra y al final la sigmática.

Su aspecto es semejante a alguno de los ejemplos mostrados por Agustín Millares (13) y Josefina Mateu (14).

\section{8.- La ornamentación}

El conjunto de iniciales iluminadas del Códice de Elche, pese a su evidente falta de calidad, se inscribe, plenamente, en el denominado estilo franco-gótico, que se impone en los territorios de la Corona de Aragón en las postrimerías del siglo XIII y permanece como base de los iluminadores a lo largo del siglo XIV.

A su influjo corresponde en el códice, de una parte, la tendencia al recuadro simple, es decir, a concebir la letra como inscrita en un marco geométrico del que sus rasgos esenciales, al modo de las grandes iniciales góticas, nunca se salen. de otra, la tendencia a destacar los trazos más característicos mediante contrastes de color - en este caso rojo y azul -, de tal modo que resulte claramente identificable el signo. Elementos procedentes de este estilo son, también, el relleno interno de la letra mediante estilizaciones geométricas, y la decoración a base de bandas.

Asímismo, es de destacar que la falta de calidad de los iluminadores ha evitado sus rasgos más característicos: bandas y enladrillados geométricos, como fondo, al modo del Cartulario de Elna (Biblioteca Municipal de Perpiñán) o los Capbreus de Clayra y Tautavel (Archivo Departamental P. O. Perpiñán). Por contra, las decoraciones, aprovechando los ángulos entre el contorno de la letra y un recuadro, pertenecen al tipo del sacramentario de San Cugat y del Códice de Durandum de Vich (15). Más cercano, geográficamente, es el Fori et privilegia Valentiae, en el que encontramos unas iniciales en las que se han empleado las palmetas y los dibujos geométricos (16).

No obstante, aparecen en la iluminación ligeros rasgos del estilo italogótico, cuya aparición en la Corona de Aragón, se centra en el siglo XIV, a consecuencia, esencialmente, de las relaciones establecidas con la universidad de Bolonia y, sobre todo, por la abundante importación de códices jurídicos. Los iluminadores de este códice conectan con el estilo de un modo mínimo, pero evidente en la tendencia a abandonar las decoraciones geo- 
métricas, sustituyéndolas por otras de carácter vegetal, aunque impregnadas del sentido caligráfico dominante en el mismo, y en la presencia de elementos geométricos de claro sentido arquitectónico: circulos, bolas, etc.

Su sentido administrativo y, por tanto, el que las iluminaciones sean obras más de escribanos que de verdaderos iluminadores, hace que las tendencias pictóricas queden muy veladas en una escuela que se halla ya utilizando verdaderos pintores al modo de los miniaturistas. No obstante, su presencia es la que no lleva, entre otros aspectos, a datar su realización en la primera mitad del siglo XIV.

Asímismo, es de señalar la existencia de varias manos, de las que al menos son de destacar dos. La primera, la de mayor calidad y de características, claramente, francogoticas, vendría representada por la inicial I, del folio $98^{\vee}$, la única en la que, al modo de los grandes iluminadores, el recuadro geométrico se intregra en el propio cuerpo de la letra que, sobre una estructura muy simple, una serie de bandas paralelas, cerradas en su parte superior, organiza una amplia decoración de filacterias, de las que las exteriores son decoradas con una sucesión de escudos y, la otra, por un mensulado, en tanto, las exteriores se quiebran en formas curvas con una doble decoración de formas geométricas, y estilizaciones vegetales e, incluso, un escudo invertido. En la parte inferior, las filacterias fingen flecos de bandoleras de ragos caligráficos, en tanto combina, predominando rojo y azul, colores no muy usuales como el amarillo plata o el verde.

La segunda, la $\mathrm{N}$ del folio 81 , que es en todo el códice la que presenta mayores influjos del estilo italogótico. Un recuadro, en cuyo interior se inscribe la letra, y en el que los ragos caligráficos se conciben en rojo y con una decoración geometrizante francogótica, pero cuyos huecos son decorados con estilizaciones de origen floral.

Como señala Villalba Dávalos (17), para la miniatura valenciana del siglo XIV, en el Libro de Privilegios de Elche encontramos influencias francesas e italianas, más o menos amortiguadas por la ejecución de un artista, posiblemente, local.

\section{9. -La encuadernación}

Este libro de privilegios fue encuadernado en el siglo XIX, en 1891, como indica la cubierta posterior y fue, entonces, cuando se debieronn reali- 
zar los cambios de orden en la foliación, situando, come hemos indicado, los folios número 23 y 22 entre el $6^{\vee}$ y 7 .

Se trata de una encuadernación de piel marrón sobre cartón y que presenta una cubierta anterior muy historiada y una posterior más sencilla. Aquella, se divide en cuatro rectángulos, efecto producido por la orla y la cruz que divide el espacio. En ambos elementos la decoración es la misma, follaje y bustos alternados, que parece representar los señores que tuvo Elche. En las esquinas de la orla y en el centro de cada uno de los lados, al igual que en el centro de los rectángulos y de la cubierta, representaciones heráldicas.

Hemos realizado un esquema de la cubierta enumerando los escudos según el número que llevan: $1^{\circ}$, en el centro, con tonos de rojo, una reproducción del sello usado por el "consell" ilicitano y que es semejante al actual escudo municipal; $2^{\circ}$, un castillo, simbolizando a astilla; $3^{\circ}$, un león, representando a León; 4ํㅡㄹ el emblema de Barcelona, ciudad que tuvo enfeudada Elche; $5^{\circ}$, la representación de la ciudad de Valencia; $6^{\circ}$, las armas de Aragón; $7^{\circ}$, el campo del escudo está ocupado por nueve granadas, acaso quiera significar Granada; $8^{\circ}$, las armas de Portugal; $9^{\circ}$, nuevamente, Castilla; $10^{\circ}$, otra vez León; $11^{\circ}$, el escudo está ocupado por una corona imperial, ¿alusión a la época de Carlos V?; $12^{\circ}$, Galicia; $13^{\circ}$, unas cadenas, símbolo de Navarra (Lam. 18)

En la cubierta posterior, en el centro un rectángulo con la siguiente leyenda:

Este privilegio que contiene / la copia de los privilegios / concedidos a Elche por los / Reyes y Príncipes que le poseyeron, lo adquirí por / donación de don Ramón Mira-llles, que lo compró en una al-/ moneda sumamente estro-/peado. Mi infortunado her-/ mano Aureliano dispuso su actual reencuadernación / cuya pureza no he sabido respetar, pues que he llena-/ do su cubierta de borrones. /Perdona hermano querido esta profanación, y tu curio-/ so investigador no mires en ella mas que el ardien-/ te deseo de realzar este te-/ soro histo(ri)co. Elche dicte 1891 / Pedro Ibarra Ruiz.

En la cubierta anterior, sobre la losange que representa a Valencia, se puede leer en las barras - en la zona amarilla: Pedro Ibarra y Ruiz 1891 / lo dib(ujó) / en Elche / 


\section{2.- HISTORIA DEL MANUSCRITO}

Muy poco conocemos de las vicisitudes del Libro de Privilegios de Elche, tan sólo que allá por el siglo XIV fue encargada su elaboración por parte del "consell" ilicitano, quizá a escribanos e iluminadores locales, sin que podamos precisar más.

En una época indeterminada, el libro, dejó de pertenecer al consistorio ilicitano, siendo adquirido en el siglo XIX por Ramón Miralles, que lo había comprado en una almoneda, aunque muy estropeado. Posteriormente, lo regaló a don Aureliano Ibarra y a su muerte pasó a ser propiedad de su hermano Pedro. Este le colocó las cubiertas actuales y tras su defunción, como todos los documentos y manuscritos que había ido recuperando a lo largo de su vida, los cedió al Archivo Municipal de Elche, donde en la actualidad se conserva.

\section{3.- BIBLIOGRAFÍA}

Uno de los motivos que nos llevó a ocuparnos de este libro de privilegios fue, precisamente, el hecho de que hasta el momento no se le hubiera hecho un estudio en profundidad, además de realizar su edición.

Ya hemos dicho que algunos de sus documentos han sido ya publicados por Torres Fontes (18), dentro de la Colección de Documentos Inéditos para la Historia de Murcia, así como por Juan Manuel del Estal (19). Con anterioridad, Gisbert Ballesteros y Pedro Ibarra habían hecho referencia a algunos de sus documentos (20).

El único trabajo que conocemos, el de Rosa M $M^{a}$ Blasco (21), estudia sólo los sistemas de datación usados en los diferentes documentos que componen el Libro de Privilegios y no aborda el estudio de forma global. 



\section{NOTAS}

(1) J.M. DEL ESTAL, Documentos inéditos de Alfonso $X$ el Sabio y el infante su hijo Sancho, Alicante, 1984

..., El reino de Murcia bajo Aragón (1269-1305). Corpus Documental l,Alicante, 1985

E. GISBERT BALLESTEROS, Historia de Orihuela, Orihuela, 1901-1903, 3 vols.

P. IBARRA, Historia de Elche, reed. Alicante, 1985

J. TORRES FONTES, Documentos de Alfonso X el Sabio, Murcia, 1963

..., El siglo XIII, Murcia, 1969

..., Fueros al reino de Murcia, Murcia, 1973

..., Privilegios de Sancho IV, Murcia, 1980

(2) R. Mํㅡㄹ BLASCO, "Notas sobre la datación cronológica en Castilla y Aragón a través de la colección de privilegios reales del Códice de 1523", en Item 4 (Alicante, 1978) p. 103-108

(3) La edición del Libro de Privilegios, esta en vías de publicación por parte del Consell de Cultura Valenciano y llevará una breve introducción.

(4) Los aspectos diplomáticos aparecerán en el próximo número de esta revista.

(5) A. PETRUCCl, La descrizioni del manoscritto. Storia, problema, modelli.Roma, 1984, p.77-86

(6) A.M.E., Códice, fol. $77^{\vee}$ y 78

(7) A.M.E., Códice, fol.: 113 "...los quals son estats tornats et translladats a la fi de un llibre o quern de pergami del consell de Elix...

(8) A.M.E., Códice, fol. 113

(9) A.M.E., Cabildos, 1523

(10) G.I. LIEFTINCK, "Pour une nomenclature de l'écriture livresque de la période dite gothique", en Nomenclature des écritures livresques du IX au XVIte, Paris, 1953, p. $15-30$

A. CANELLAS, Exempla scripturarum in usum scholarum, Zaragoza, 1974, 2 vols. J. MATEU IBARS, Collectanea Paleografica de la Corona de Aragón, Barcelona, 1980-1991, 2 vols. F. GASPARRI, "La terminologie des écritures", en CoIloqium des Comite Internationale de Paleographie, Munich, 1981, p. 29-32

M. C. ALVAREZ, "Escritura latina en la plena y baja Edad Media: la llamada "Gótica libraria", en Historia, Instituciones y Documentos, 12 (Sevilla, 1986), p. 1-34 Me refiero a los estudios aparecidos en: ALFONSO X, Especulo, edición y análisis crítico de Gonzalo Martínez Díez, con la colaboración de José Manuel Ruiz Asencio. Avila, 1985, p. 65-76 
..., Fuero Real, edición y análisis crítico por Gonzalo Martínez, con la colaboración de José Manuel Ruiz Asencio y César Hernández Alonso. Avila, 1988, p. 140-152

W. MEYER, Die Buchstaben-Verbindungen der sogenannten gothischan Schrift. Berlin, 1897

S. ZAMPONI, "Elisione e sovrapposizione nella littera textualis", en Scrittura e civiltà, 12 (1988) p. 135-139

(11) A. MILLARES CARLO, Tratado de ..., lam. 187

(12) M. USON SESE, Contribución al estudio de la cultura medieval aragonesa. La escritura en Aragón del siglo XI al XVI, Zaragoza, 1944, p.

F.C. CASULA, "Alcune note sulla "letra aragonesa" del secolo XIV" en Annali delle Facolta di Lettere, Filosofia e Magisterio dell'Università di Cagliari, XXX (1967) p. 30-45

(13) A. MILLARES CARLO, Tratado ..., lam. 244

(14) J. MATEU IBARS, Collectanea..., lams. 119, 125, 135 y 139

(15) J. DOMINGUEZ BORDONA, "La miniatura", en Ars Hispanie, vol. XVIII, Madrid, 1958, p. 136 y 137

(16) A. VILLALBA DAVALOS, La miniatura valenciana de los siglos XIV y XV, Valencia, 1964, p.22

(17) A. VILLALBA DAVALOS, La miniatura..., p. 44 19.- Ver nota 1

(18) Ver nota 1

(19) Ver nota 1

(20) Ver nota 1

(21) Ver nota 2 
$\langle>\langle\mid\rangle\langle\rangle$

$<><>|<\rangle<><>$

$<><>\mid<p<>$

$<p<\geqslant \mid<y<\geqslant$

$<><>\mid<><>$ 
$<\geqslant<>1<><>$

$<><>1<p<p$

$<><>|<\rangle<>$

$<><>\mid<><>$

$<P<P \mid<p<>$

2 
$\langle><>\mid<\rangle<\%$

$<><\geqslant \mid<><>$

$<><>|<\rangle<>$

$<p<>\mid<p<p$

$<><>1<><?$

《! 

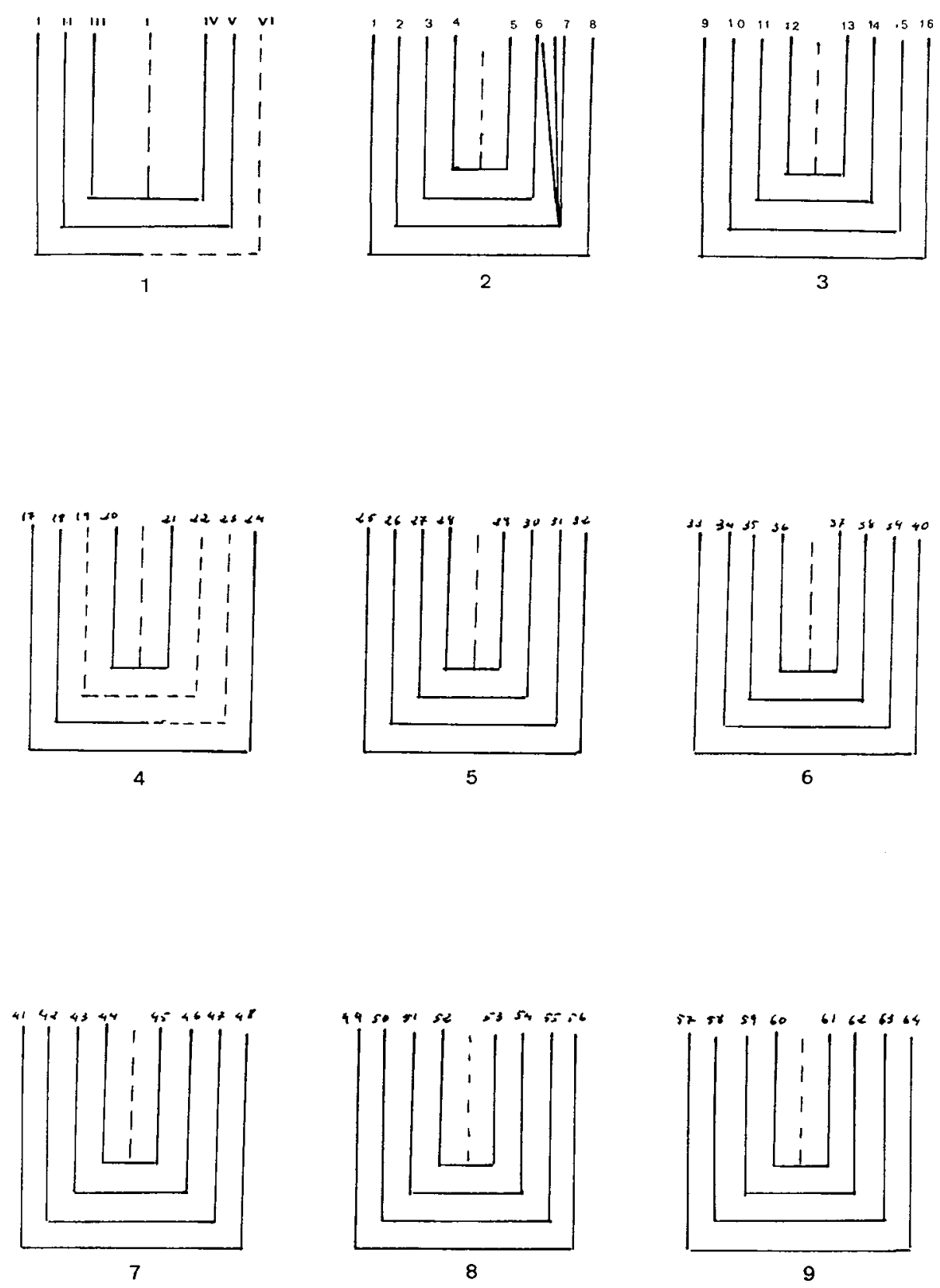

4 

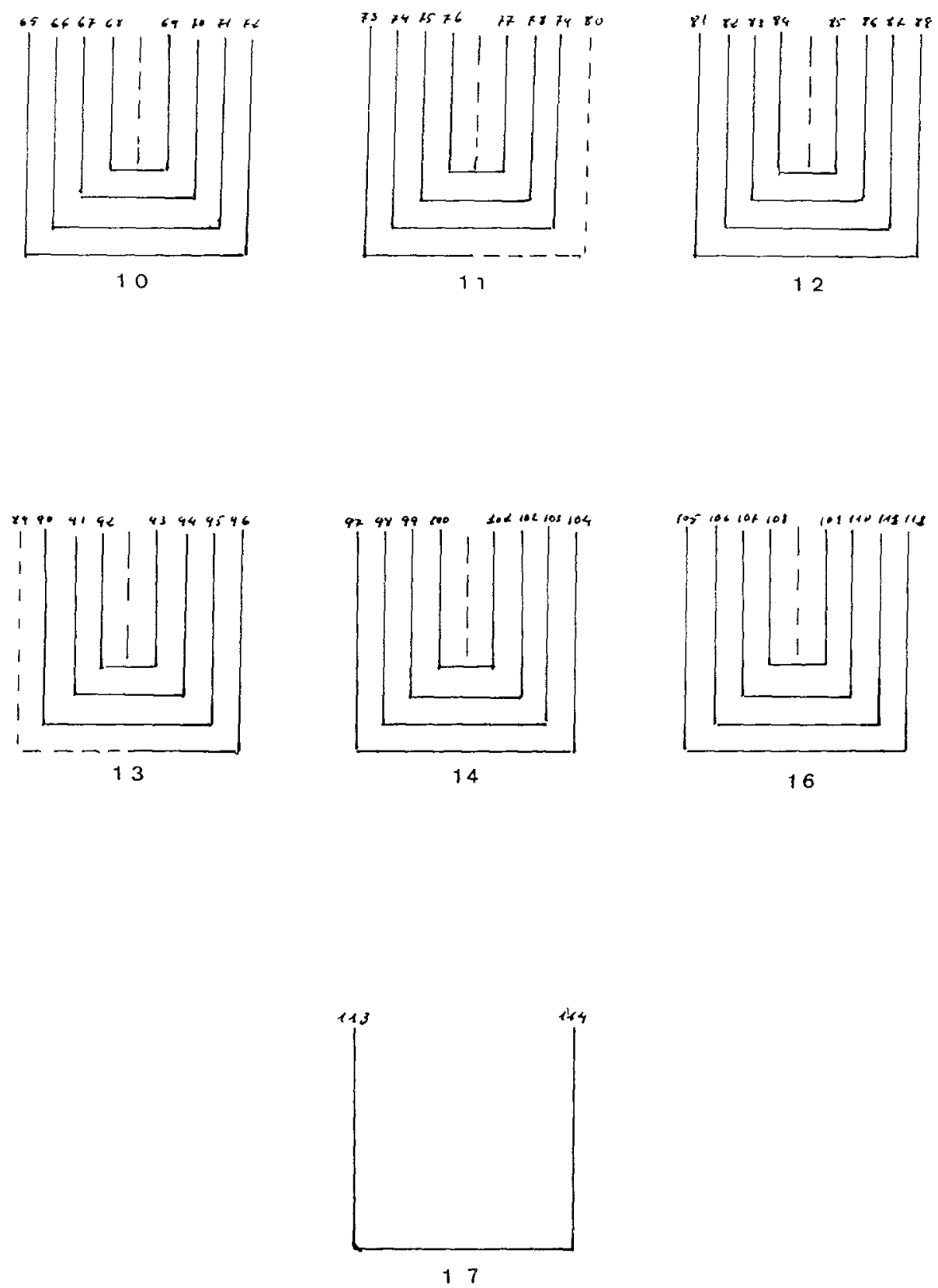

5 


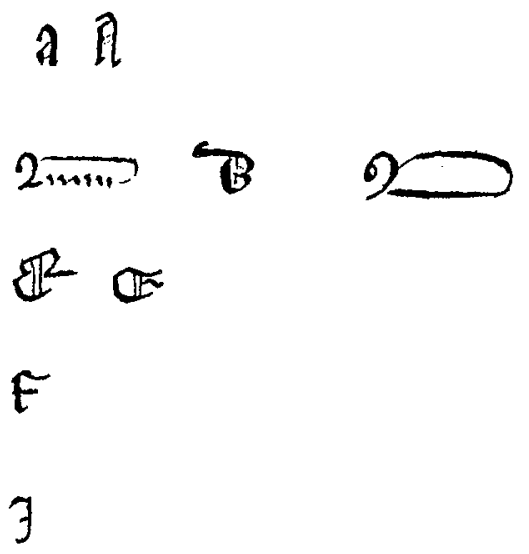

$D$ 的

p 2 구

(1)

tomeno

$\mathbb{R}$

$\not 2$

T)

$\mathcal{Y}$

ALFABETO MAYUSOULO

6 


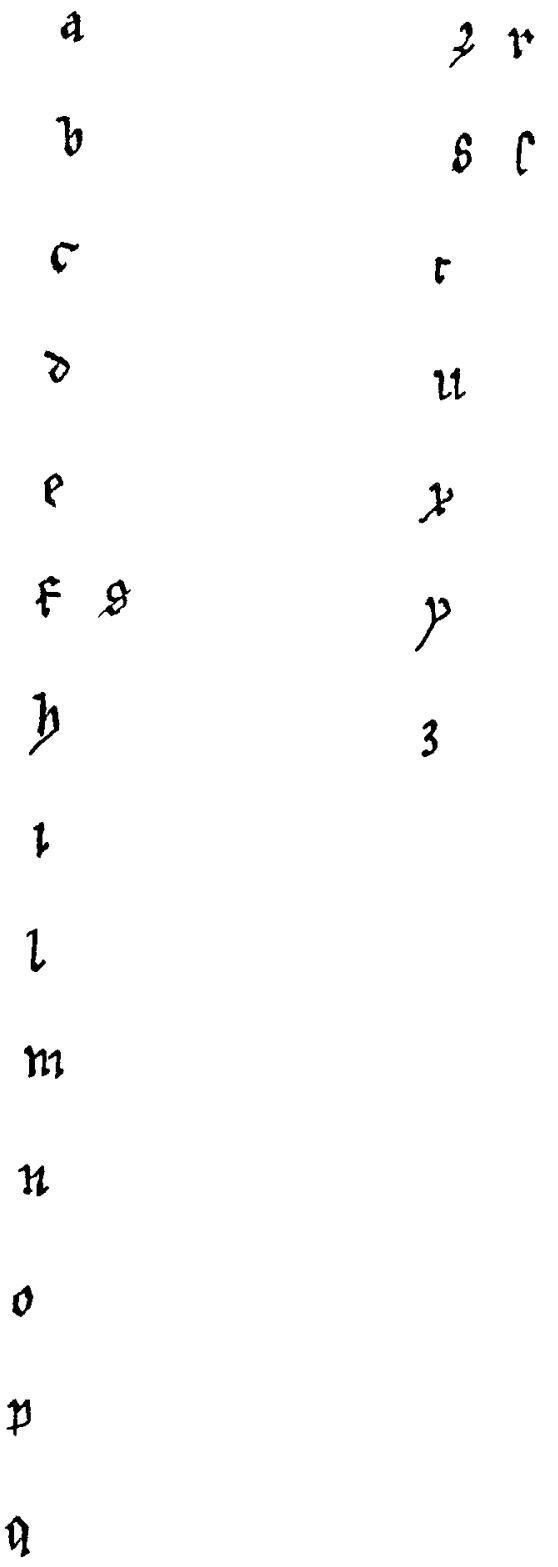

AL FABETO MINUSCULO

7 
$\mathbb{E}$

$$
10=p 0
$$

4

$$
d e=d e
$$

\section{b}

$$
\begin{aligned}
& \text { Q }=\text { or } \\
& \mathbb{Q}=s t
\end{aligned}
$$

)

IIGADURA S

$$
\begin{aligned}
& z=\theta^{t} \\
& Z=\text { her }
\end{aligned}
$$


$d$

b

e

i

c

F

$s$

h

1

$\downarrow$

171

11

0

p
9

$1+2$

is

$t$

11

$x$

y

3

ALFABETO MINUSCULO

10 


$$
q=o r \quad \text { it }=a t
$$

\section{pye Porum}

de de

\section{IIGA IURAS}

$$
\begin{aligned}
& \mathscr{H}=\text { quod }=\text { contra } \\
& \dot{y}=\text { ser } \\
& \dot{H}=\text { per } \\
& \dot{y}=\text { pro } \\
& \dot{t}=\text { ver } \\
& \dot{t}=\text { tur } \\
& z=\text { et } \\
& \psi=\text { mum } \\
& \text { SISTEMA ABREV IATIVO }
\end{aligned}
$$

\section{1}




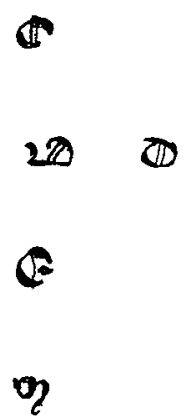

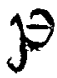

z

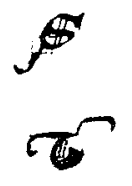

ALFABETO MAYUSCULO

12 

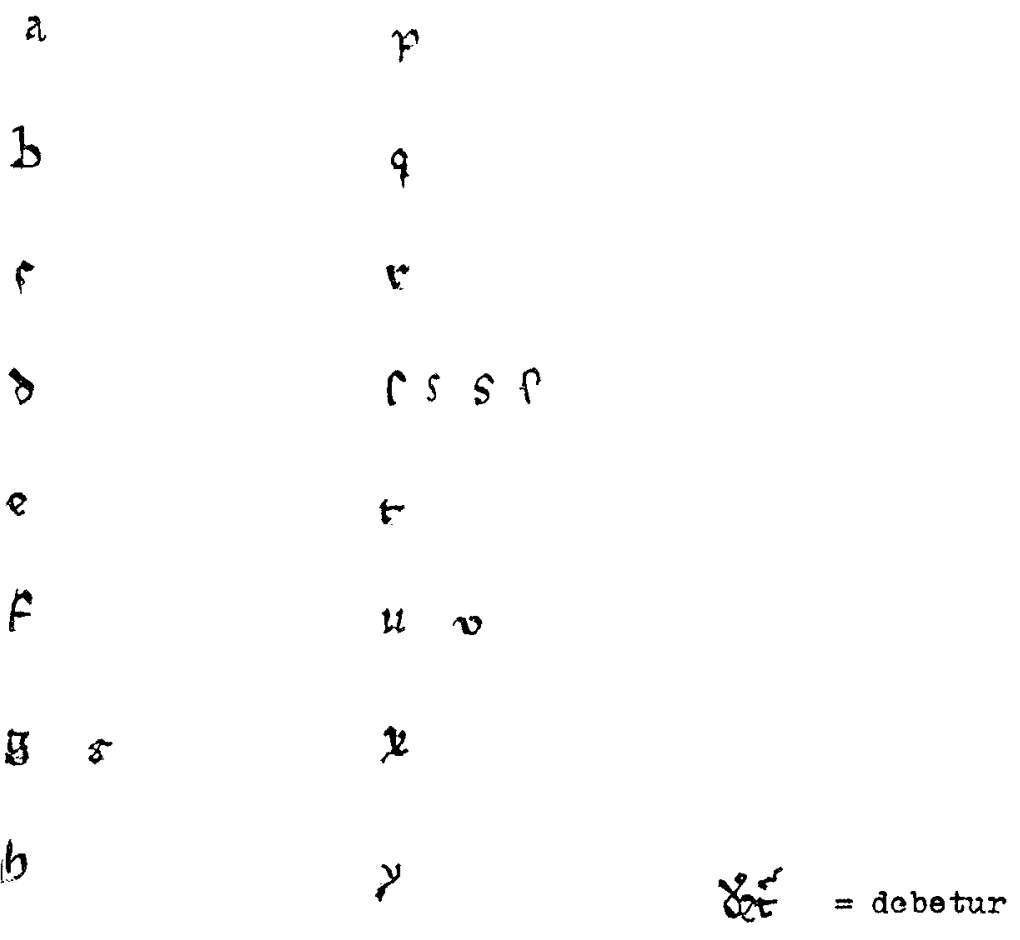

$\mathfrak{l}$

ho

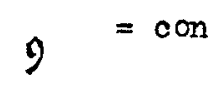

$\mathfrak{l}$

$\mu=\operatorname{mam}$

il = ver

IM

$\mathfrak{n}$<smiles>C[Te]=[Ca]</smiles>

$Q=$ que

$p$ 


\section{5}

1

1

4)

ALFABETO MAYUSCULO

15 


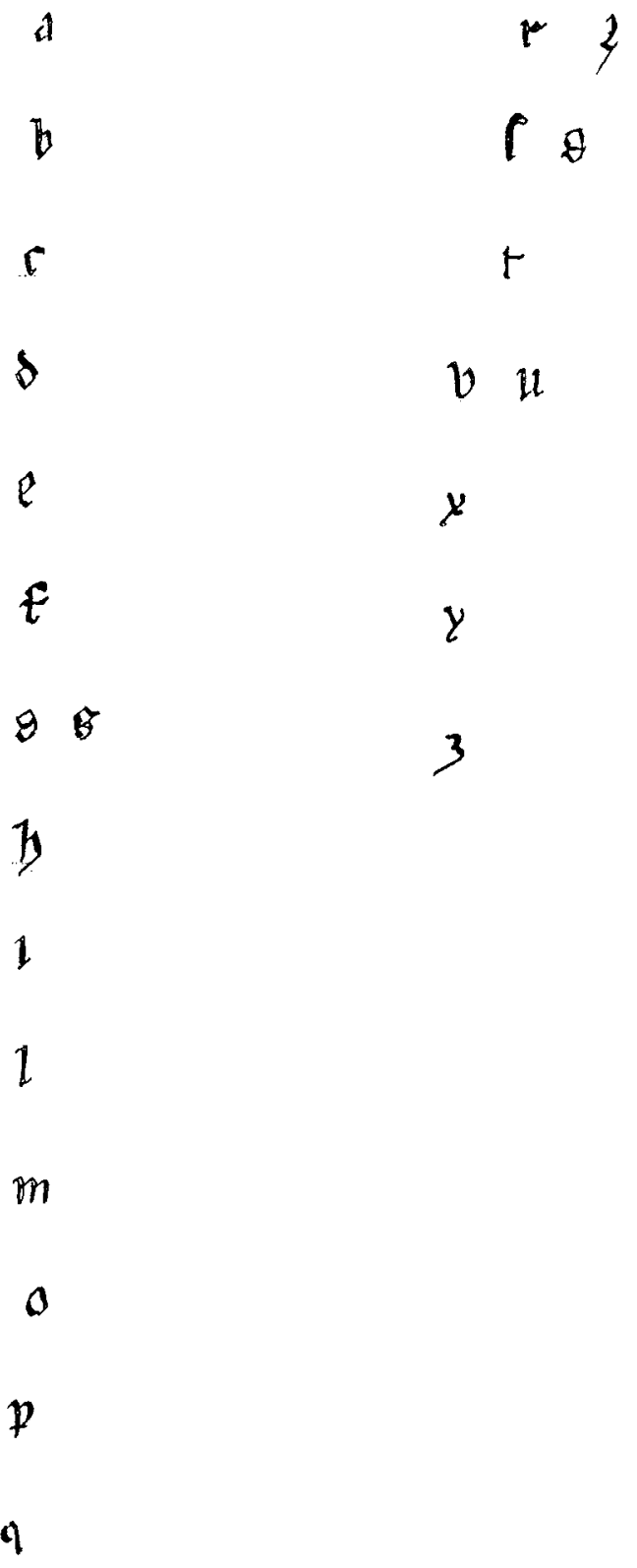

ALFABETO MINUSCULO

16 


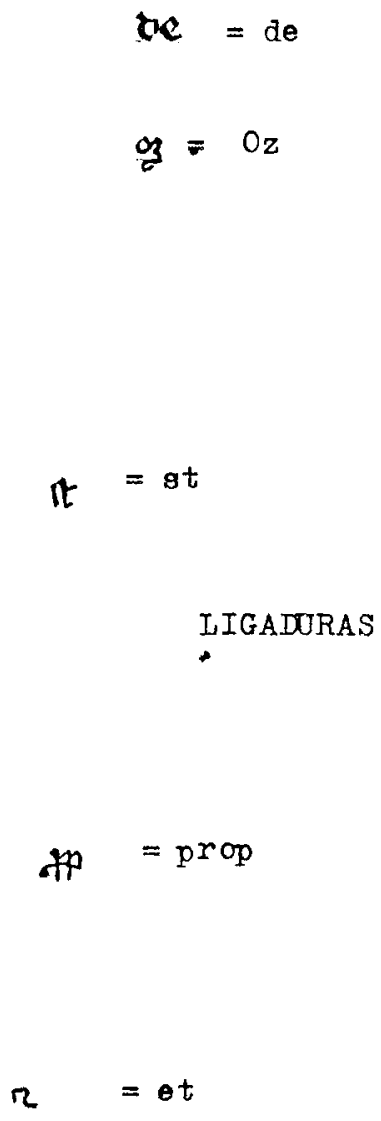




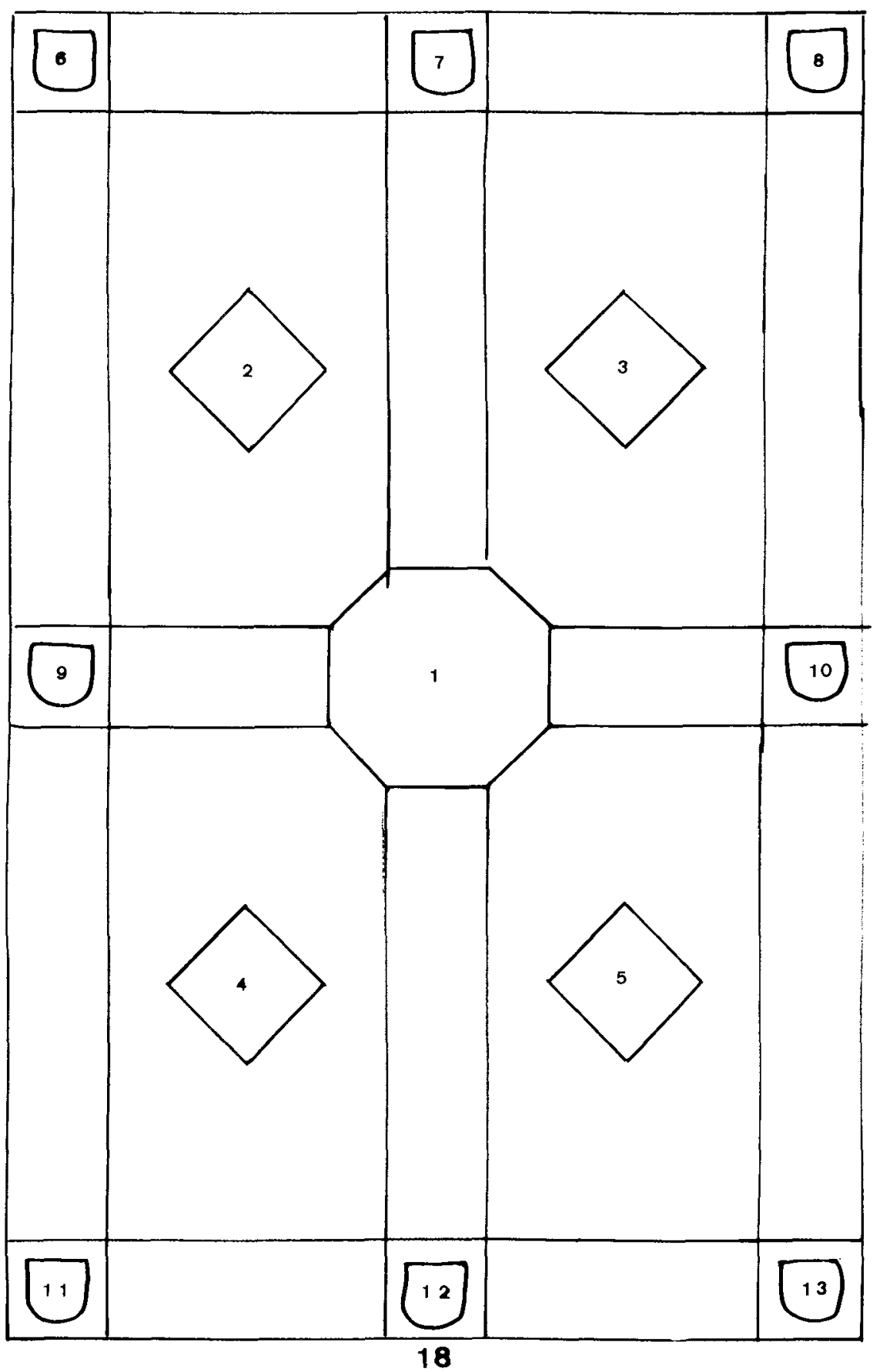

